\title{
QUAND LA SOCIOLOGIE DES SCIENCES SE SAISIT DE LA SOCIOLOGIE DE L'ART
}

\author{
Emmanuelle Sebbah*
}

\begin{abstract}
Résumé: Si, classiquement, la sociologie des sciences est envisagée comme l'étude des activités relevant des sciences de la nature, c'est dans le cadre d'une sociologie des sciences humaines et sociales que l'on souhaite insérer nos propres recherches, et étudier la constitution de la sociologie de l'art en France, comme sousdiscipline distincte des autres champs d'investigations de la sociologie et distincte des autres disciplines traditionnellement en charge de son objet: l'esthétique et l'histoire de l'art. C'est sur le premier moment du processus de "disciplinarisation" de la sociologie de l'art que nous nous arrêterons, à savoir son insertion au sein du projet durkheimien.
\end{abstract}

Mots-clés: Sociologie des sciences, histoire de la sociologie, discipline, disciplinarisation, communauté, Année sociologique, Emile Durkheim.

\section{Sociologie des sciences et sociologie des sciences humaines et sociales}

Classiquement, la sociologie des sciences est envisagée, souvent implicitement, comme la spécialité de notre discipline qui prend pour objets les sciences de la nature - de la même manière que la notion de sciences renvoie communément aux sciences de la nature. C'est en tout cas la manière dont elle est couramment présentée dans les manuels consacrés à la question, lesquels associent fréquemment le développement des techniques au traitement des sciences dites exactes. Sans doute la paternité des recherches dans le domaine classiquement attribuée à la thèse que Robert K. Merton

Affiliée ou GEPECS, Université Paris 5 - René Descartes.

Artigo recebido em 16 ago. 2005; aprovado em 12 out. 2005. 
consacra en 1938 à l'émergence et au développement des sciences et de la technique en Angleterre au XVII ${ }^{\mathrm{e}}$ siècle en Europe a-t-elle contribué à cette réduction présumée de l'objet. Pourtant, ce procédé ne va pas de soi, et des travaux, de plus en plus nombreux, d'orientations très diverses, témoignent d'un intérêt sociologique des sociologues pour leur discipline, ou, plus généralement, pour les sciences humaines et sociales. Sans pour autant procéder à une transposition mécanique des outils, théories et méthodes que propose la sociologie des sciences, l'attention est tantôt portée sur la constitution d'un "champ" disciplinaire ou d'une École, tantôt sur des contextes de production ou de réception d'une discipline, d'une théorie, d'un courant ou d'un auteur; les exemples sont nombreux. ${ }^{1}$ La recherche que l'on mène et que l'on souhaite ici évoquer trouve sa place au sein d'un tel questionnement et concerne la constitution de la sociologie de l'art en France. Autrement dit, la sociologie de l'art constitue l'objet de la recherche, et non le cadre à l'intérieur duquel celle-ci viendrait s'inscrire.

Les sociologues des sciences le savent bien, cette "sociologie de la sociologie - de l'art" n'a ni vocation à discuter la scientificité de son objet - la sociologie de l'art - ni la pertinence ou la validité des thèses qui y sont défendues ou des résultats qui y sont proposés. Le travail poursuivi consiste plutôt en une compréhension du processus historique de "disciplinarisation" à l'œuvre dans ce domaine, ainsi que nous allons le voir.

\section{Rendre compte du processus de "disciplinarisation" de la sociologie de l'art}

L'on souhaite comprendre comment la sociologie de l'art s'est historiquement constituée en discours relativement autonome, c'està-dire distinct d'une part des autres domaines d'investigation de la discipline sociologique, et d' autre part des disciplines traditionnellement en charge de son objet, à savoir l'histoire de l'art et l'esthétique. Il s'agit donc d'identifier les étapes successives de constitution du domaine - c'est-à-dire les différentes configurations institutionnelles 
et cognitives, parce qu'une discipline se définit simultanément par les institutions qui la portent et par les théories développées au sein de ces institutions.

Il nous faut toutefois garder à l'esprit que dans bien des cas, le travail de segmentation disciplinaire et d'autonomisation des spécialités ne s'est effectué que très progressivement, et que les frontières cognitives mais aussi institutionnelles ne se sont affirmées que très tard. Jusque très récemment en effet, les frontières disciplinaires étaient fréquemment franchies, et la "logique de l'objet" a longtemps prévalu sur la "logique disciplinaire" (Mucchielli, 1998, p. 9 notamment). Aussi mesure-t-on les irrémédiables réductions que l'usage, en sociologie, de la notion de discipline impose. ${ }^{2}$ De ce fait, Laurent Mucchielli (1998) invite à pratiquer une "histoire $d u$ processus de disciplinarisation", plutôt que de "réduire [les æuvres] à une dimension disciplinaire", ce qui reviendrait à les mutiler.

Ce processus - dans une certaine mesure inachevé d'émancipation institutionnelle et intellectuelle s'est assorti d'un travail de segmentation de la discipline en spécialités. En effet, l'histoire de la sociologie nous enseigne que sa construction est profondément marquée par une spécialisation des recherches autour de domaines, d'objets toujours plus définis constituant autant de sous-disciplines, même si ce cloisonnement est tout relatif et son dépassement souvent revendiqué. ${ }^{3}$ Il nous paraît même impossible d'évoquer le processus de "disciplinarisation" sans évoquer l'organisation interne - le découpage de la sociologie en spécialités - en même temps que de la construction des frontières externes de la sociologie. L'histoire de la sociologie de l'art témoigne remarquablement, nous semble-t-il, de cette interdépendance entre l'autonomisation de la discipline et les découpages qu'elle connaît en son sein.

\section{Parce que “l'art et la sociologie ne font pas bon ménage”...}

Cette constitution du domaine s'étant donc nécessairement effectuée dans un rapport direct - ou en opposition directe - avec 
les discours jusqu'alors dominants sur l'art, celui de l'esthétique et celui de l'histoire de l'art, la singularité de la sociologie de l'art naissante nous a amenée à formuler une série d'hypothèses dont nous restituons ici le point nodal.

Nous avions en effet acquis la conviction, au cours de nos recherches, que ce processus de "disciplinarisation" du domaine avait été particulièrement malaisé en raison de la nature même de l'objet rencontré. S'il ne s'agissait pas pour les premiers sociologues français de se substituer à leurs prédécesseurs ou à leurs contemporains historiens d'art ou philosophes esthéticiens, l'abord proprement sociologique de leur objet n'allait pour autant pas de soi. Précisément, le mythe contre lequel s'érigeront les sociologues de l'art tout au long de son développement consiste justement en une conception, parfois implicite, dominante au XIX siècle, d'un artiste possédant, sinon un don, un talent particulier, faisant de lui un créateur à la fois original et inédit et répondant aux exigences stylistiques et académiques de son époque. C'est le mythe de l'artiste en " régime de la vocation artistique", "exutoire à la tension entre l'exigence de la conformité, synonyme de qualité académique, et l'exigence de rareté, synonyme d'originalité" (Heinich, 1998). Cette représentation de l'artiste exclut, de manière générale, la réflexion sur les éventuelles déterminations sociales de sa vocation artistique ou de son génie, ou en tout cas, leur résiste très largement.

De la même manière que le mythe du savant isolé ne permet pas de rendre compte de l'activité scientifique, ni de comprendre comment se fait la science, la représentation romantique de l'artiste n'autorise a priori pas le recadrage sociologique. Il est évident que dès lors que l'on met en évidence tout une série de mécanismes sociaux qui jouerait un rôle dans l'accréditation de tel artiste ou de telle œuvre, de tel courant ou de tel type d'art, l'opération entreprise est, de fait, réductionniste. Il est d'ailleurs plutôt courant de trouver, dans les propos des sociologues de l'art, un discours sur les valeurs antagoniques qui sont celles de la discipline étudiante et de l'objet étudié, la sociologie et l'art. Comme le dit Bourdieu (1980) dans un 
célèbre article, souvent repris et cité par des sociologues de l'art, "la sociologie et l'art ne font pas bon ménage":

La sociologie et l'art ne font pas bon ménage. Cela tient à l'art et aux artistes qui supportent mal tout ce qui attente à l'idée qu'ils ont d'eux-mêmes : l'univers de l'art est un univers de croyance, croyance dans le don, dans l'unicité du créateur incréé, et l'irruption du sociologue, qui veut comprendre, expliquer, rendre raison, fait scandale. Désenchantement, réductionnisme, en un mot, grossièreté ou, ce qui revient au même, sacrilège: le sociologue est celui qui, comme Voltaire avait chassé les rois de l'histoire, veut chasser les artistes de l'histoire de l'art.

C'est ce que relèvera également Nathalie Heinich (1998) dans Ce que l'art fait à la sociologie:

L'individuel opposé au collectif, le sujet au social, l'intériorité à l'extériorité, l'inné à l'acquis, le don naturel aux apprentissages culturels : le domaine de l'art est par excellence celui où s'affirment les valeurs contre lesquelles s'est constituée la sociologie.

\section{Un défi lancé aux sciences sociales}

Parce que l'objet étudié, ou plutôt sa représentation, semble donc résister aux cadres épistémologiques de la discipline étudiante, l'étude de l'art pouvait représenter pour les fondateurs de la sociologie française et pour leurs successeurs un véritable défi, analogue à celui qu'avait pu représenter l'appréhension du suicide par exemple. Ce rapport si particulier entre la discipline et son objet une fois envisagé, il est possible de comprendre les différents programmes de recherche qui se sont succédés dans l'histoire de la sociologie de l'art comme autant de propositions de résolution du problème posé.

La question artistique, plus que toute autre, nous le montrons plus loin, incarne donc ce défi lancé aux sciences sociales en ce qu'elle les oblige à la fois à édifier des frontières et à les franchir ; les transgresser parce qu'elles doivent nécessairement se nourrir des travaux issus des disciplines qui prennent classiquement en charge ces questions; les édifier pour attester qu'elles peuvent traiter ces 
questions comme elles abordent d'autres faits sociaux. Il nous reste à présent à nous demander, en interrogeant la place qu'occupait la question artistique chez les fondateurs, la manière dont si ce défi a été initialement relevé.

\section{L'Année Sociologique et l'art}

Les colonnes de la première série de l'Année Sociologique, ${ }^{4}$ organe du groupe durkheimien dont on sait le rôle décisif dans la constitution de la sociologie française, constituent un lieu d'observation idéal et privilégié, en ce que s'y trouve matérialisée l'existence de la sociologie française et médiatisé le contenu du savoir sociologique naissant. Parfois analysée et dépeinte comme remplissant les fonctions d'un véritable centre de recherche (cf. Clark, 1968, p. 72-91), la revue est l'outil de compréhension de la naissance de la discipline sociologique, "l'instrument de définition des contours et des frontières de la sociologie" (Mucchielli, 1998, p. 253). On verra que si c'est l'intégralité des sciences humaines qui se trouve saisie par les visées impérialistes de la sociologie, la question artistique n'y échappe alors pas, malgré la place de fait résiduelle qui lui est réservée. Cette incursion systématique de la sociologie dans différents domaines de connaissance trouve son aboutissement dans l'appropriation ou plutôt l'annexion de leur objet et l'affirmation de l'originalité de l'approche spécifiquement sociologique.

Sans vouloir conférer à la sphère artistique une place centrale dans l'horizon des préoccupations de Durkheim ou de ses proches collaborateurs, "sans aller jusqu'à procéder à une inversion de signes qui attribuerait à la marginalité d'une question le prestige symptomatique d'un pensé ou d'un impensable théorique" (Menger, 2001, p. 313), nous souhaitons en premier lieu insister sur le défi que pouvait ou qu'aurait pu représenter le traitement de la question artistique dans l'entreprise impérialiste de l'équipe de l'Année, et plus particulièrement pour son chef de file. L'art peut se présenter, nous l'avons déjà relevé, à l'observateur, comme un objet ontologiquement réfractaire à l'analyse sociologique, et ainsi constituer 
l'objet rêvé d'une mise à l'épreuve du postulat durkheimien. En effet, création qui procède en apparence d'une subjectivité, strictement individuelle, l'œuvre artistique ne s'inscrit pas aisément dans les cadres épistémologiques d'une discipline qui pointe la détermination collective des créations individuelles. Aussi aurait-il été ambitieux, à contrepied du sens commun, de montrer que l'acte créatif et les œuvres artistiques d'apparence strictement individuels sont justiciables d'une analyse sociologique, parce qu'ils sont profondément déterminés par la réalité collective. Le choix de la spécialité ici évoquée, l'art, n'est donc pas anodin. Il est à plus d'un égard problématique.

\section{Le plan de classification de l'Année}

Il est important de rappeler ici succinctement en quoi consista le projet du groupe durkheimien, et d'insister sur le fait qu'il témoigne, malgré les évidentes incursions des uns et des autres dans des domaines connexes, d'une volonté de supplanter la "logique de l'objet " au profit de la "logique disciplinaire".

A la fin du XIX siècle, la discipline sociologique n'existe pas, et il est impératif de la doter à sa naissance d'une "légitimité scientifique" et d'une "légitimité institutionnelle". ${ }^{5}$ Dans ce dessein, Durkheim, entouré de ses proches collaborateurs, se consacre à la création d'une revue, analogue en plusieurs points à celles qui existent déjà à l'époque. "Si le titre de l'Année sociologique n'est qu'une reprise, ses principales caractéristiques ne constituent pas davantage une innovation. Les Années présentant les principales productions du savoir étaient très en vogue depuis quelque temps. Citons entre autres avec leur date de fondation l'Année artistique (1878), l'Année médicale (1878), l'Année archéologique (1879), l'Année épigraphique (1888) (...), l'Année philosophique et l'Année psychologique" (Besnard, 1979, p. 10). Les enjeux disciplinaires liés à la fondation de ce type de publication sont majeurs, on le sait. En dehors de l'existence de réseaux ${ }^{6}$ d'acteurs dont témoignent la naissance et la survie d'une revue (éditeur, équipe d'auteurs, etc.), c'est l'organisation de la discipline sociologique "autour de champs 
particuliers, qui seront autant de catégories de classement de la matière sociologique" "q que l'analyse des colonnes de la première série de l'Année permet d'appréhender. Seulement, si la revue a pour vocation dès sa création, de présenter les principales productions de la discipline, rappelons que la littérature sociologique n'est quant à elle pas suffisamment abondante pour alimenter ses colonnes. L'intention de l'équipe durkheimienne est à cet égard des plus claires:

L'Année sociologique n'a pas pour seul objet ni même pour principal objet de présenter un tableau annuel de l'état où se trouve la littérature proprement sociologique. Ainsi circonscrite, la tâche serait trop restreinte et de médiocre utilité ; car les travaux de ce genre sont encore trop peu nombreux pour qu'un organe bibliographique spécial soit nécessaire aux travailleurs. Mais ce dont les sociologues ont, croyons-nous, un pressant besoin, c'est d'être régulièrement informés des recherches qui se font dans les sciences spéciales, histoire du droit, des mœurs, des religions, statistique morale, sciences économiques, etc., car c'est là que se trouvent les matériaux avec lesquels la sociologie se doit construire. Répondre à ce besoin, tel est, avant tout, le but de la présente publication. (Introduction à l'Année sociologique, t. I, 1896-1897)

Cette déclaration d'intention invitant à emprunter aux "sciences spéciales" permet d'appréhender plus justement le plan de classification de la revue. La première série de l'Année sociologique est constituée de douze volumes dont la publication s'étale de 1898 à 1913, tous publiés du vivant de Durkheim qui en tient la direction. Chaque livraison est censée recenser les travaux de l'année précédente. Ainsi, la première livraison, de 1898, inventorie et commente les travaux parus en 1896-1897, la deuxième recense ceux des années 1897-1898, et ainsi de suite. La revue fait par ailleurs l'objet d'une division essentielle en deux parties, la première partie de la revue consacrée aux "mémoires originaux" ayant été sacrifiée au bénéfice de la seconde, plus importante quantitativement, consacrée aux "analyses et notices bibliographiques". C'est à cette partie que nous consacrons toute notre attention, d'une part parce qu'avec “l'émergence $d u$ système des disciplines universitaires en sciences humaines dans les années 1870-1880, le compte-rendu est devenu un élément à part entière de la 
communication scientifique", constituant parfois "une arme critique bien plus efficace que l'article de doctrine" (Mucchielli, 1998, p. 9), et d'autre part parce que c'est dans cette section de la revue que se donne à voir le plan de classification de la sociologie. En effet, le plan de classification auquel obéit la deuxième partie de chaque volume doit témoigner de la distribution des spécialités dont traite la sociologie. C'est en ceci que l'on se doit d'y porter la plus extrême attention. Aussi, Durkheim écrit-il lui-même, dans une lettre à Célestin Bouglé (datée du 6 juillet 1900) que "ces questions de classification sont importantes, car c'est la sociologie qui s'organise ainsi. Ce n'est pas rien que de mettre un peu d'ordre dans cette masse informe".

L'analyse des volumes constituant cette première série fait apparaitre, à quelques variantes près, ${ }^{8}$ une répartition entre 7 rubriques habituelles, elles-mêmes subdivisées, qui sont la sociologie générale, la sociologie religieuse, la sociologie juridique et morale, la sociologie criminelle, la sociologie économique, la morphologie sociale, et une septième section qui regroupe sous l'intitulé “Divers" plusieurs "sciences" dont la technologie, l'esthétique et la linguistique. Le tableau figurant en annexe, construit sur la base de l'observation des douze livraisons étudiées, permet d'observer l'évolution et les modifications du plan de classification de cette dernière rubrique. C'est sur celle-ci, apparemment fourre-tout et changeante, que nous allons nous arrêter.

\section{Une place marginale pour l'art...}

Une recherche que nous avions précédemment entreprise ${ }^{9}$ avait pour objectif de mesurer la place - marginale mais constante - que la sociologie naissante avait accordé à la sphère esthétique. De fait, " qui a lu les ceuvres fondatrices de la science sociale sait que le traitement des faits et des valeurs artistiques occupe une place modeste chez des auteurs comme (...) Durkheim" (Menger, 201, p. 313). Toutefois, en dépit en effet "la place marginale" de "la question esthétique "10 chez les fondateurs de la discipline, il n'en 
reste pas moins que la rubrique sociologie esthétique, constituée dès la troisième livraison de l'Année, subsista durant toute la première série de la revue.

Contrairement à ce qu'affirme Tiryakian (1979, p. 97) dans un article consacré à " la parenté sociologique entre [l']école [durkheimienne] et l'art d'avant-garde", à savoir que "la rubrique des arts [aurait] servi de terrain d'entraînement pour les nouveaux membres de l'Ecole" (p. 104) durkheimienne, nous avons insisté sur tout l'intérêt que la présence d'une telle rubrique pouvait revêtir dans le cadre du projet disciplinaire en cours, intérêt souligné à plusieurs reprises par Mauss à l'occasion des deux volumes constituant la deuxième série qui paraîtront quelques années après la guerre, et dans lesquels il reviendra sur le plan de classification de la première série de la revue.

Ainsi, dans Divisions et proportions des divisions de la sociologie, Mauss (1927, p. 181) déplorant à plusieurs reprises le caractère particulièrement imprécis, problématique, "général et insignifiant" du chapeau Divers dont sont affublées sans distinction l'esthétique, la technologie et la linguistique et le manque de proportions dont elles sont victimes, il affirme qu' "il faut le répéter bien haut, les trois groupes de faits: linguistique, technique, esthétique, tiennent, dans les systèmes sociaux primitifs ou évolués, une place infiniment plus grande que celle que nous savons leur donner". "Même nos études et la sociologie entière se ressentent de la petite quantité des travaux consacrés à la linguistique, à la technologie et à l'esthétique (...) Le peu d'attention que nous pouvons leur consacrer nous est même pénible" écrit-il encore (p. 191). Quand il évoque spécifiquement le champ des études esthétiques, il va même jusqu'à ajouter que "la grandeur de la sociologie esthétique est peut-être encore plus notable par rapport à d'autres divisions auxquelles nous semblons ajouter plus d'importance. Cette grandeur, nous n'avons jamais pu, malheureusement, la mettre en lumière" (p. 191). 
C'est toujours chez Mauss (1927, p. 178) que l'on trouve exposées les raisons de ces lacunes, dont il s'excuse. "Le défaut est bien involontaire" affirme-t-il. Il explique qu'il a manqué à l'équipe "les spécialistes de la morphologie sociale, de la linguistique, de la technologie et de l'esthétique purement sociologiques", et ajoute que "leur intervention eut changé et l'aspect même de nos études et [...] les proportions des parties mêmes de l'Année sociologique".

\section{Une unité difficilement identifiable...}

En dépit du sous-traitement réservé à la question esthétique par les fondateurs de la discipline, Mauss (1927, p. 178) insiste sur le fait que l'effort a toujours été fait de "ne la jamais perdre de vue dans l'Année". La rubrique n'est certes apparue que dès la troisième livraison de la revue, mais contrairement aux autres rubriques de la section "divers" qui connaissent des changements, sa stabilité est remarquable et une dizaine de pages y est systématiquement consacrée. C'est donc à présent le contenu de cette rubrique qu'il nous faut aborder. Avouons-le d'emblée, le domaine que recouvre la sociologie esthétique est particulièrement flou et ses contours plus qu'imprécis. "Mal délimité", on y trouve aussi bien "l'art primitif et l'archéologie mais aussi la littérature (poésie et tragédie grecque, poésie au moyen âge, etc.), [la] musique et [les] jeux", ainsi que le souligne Marcel Fournier (1987). L'observateur même le plus aguerri est bien en peine d'y trouver une quelconque unité. Citons en vrac la recension par Mauss d'un ouvrage d'histoire sur les débuts de la poésie, ${ }^{11}$ le commentaire d'un ouvrage de Bergson, Le rire: essai sur la signification du comique, ${ }^{12}$ celui d'un opus sur le sport, ${ }^{13}$ mais également des notes critiques sur des ouvrages dont les titres témoignent de prétentions sociologiques bien plus évidentes, comme un texte de Parodi sur La méthode scientifique de l'histoire littéraire ${ }^{14}$ de G. Renard, "à égale distance de la critique logique et de l'cuvre sociologique", ${ }^{15}$ ou un autre, du même auteur, à propos 
d'une Sociologia estetica, ${ }^{16}$ qui contient " un effort pour constituer la 'sociologie esthétique' qui peut être fécond ". ${ }^{17}$

Par-delà cette diversité et, à notre sens, ce manque évident de cohérence dans les choix des ouvrages retenus, la volonté d'inscrire ces recherches dans les cadres épistémologiques de la discipline naissante est bien là : "l'importance sociologique" de l'Essai sur le rire de Bergson " ne saurait [...] être niée ", ${ }^{18}$ avec l'opus sur les débuts de la poésie cité plus haut, "l'esthétique devient définitivement une branche de la sociologie ", ${ }^{19}$ et la critique est faite à l'auteur de L'œuvre d'art et l'évolution d'être "plus artiste que sociologue ", ${ }^{20}$ les exemples sont mobilisables à l'envi.

Si le dessein des membres du groupe durkheimien consiste à "opérer l'unification organique des disciplines sociales spécialisées sous l'égide de la sociologie", ${ }^{21}$ en dégageant son objet propre emprunté à chacune d'entre elles, on est ici en droit de se demander si l'éclatement apparent de la rubrique n' aurait pas été le symptôme des difficultés que la sociologie a pu avoir à composer avec la sphère esthétique. La synthèse des travaux paraît ici bien délicate, et n'est pas selon nous à imputer uniquement au manque de spécialistes de l'esthétique que peut évoquer Mauss dans ses écrits. C'est la raison pour laquelle nous allons nous demander, enfin, si les problèmes manifestes posés par l'analyse de l'art ne désigneraient pas clairement "certaines des limites, des incertitudes interprétatives et des apories rencontrées à l'origine de la science sociale", ainsi que le suggère P.-M. Menger.

\section{Art et sociologie?}

L'observation des tables de l'Année et l'analyse du contenu de la rubrique consacrée à l'esthétique permet d'établir un double constat: si l'exercice d'annexion de territoires par la transgression des frontières disciplinaires est en effet réalisé au moyen de la création et du maintien constant de la rubrique et par la diversité des disciplines et thèmes convoqués, celui qui consiste à dégager l'unité cognitive 
des travaux autour d'un questionnement unificateur paraît nettement plus laborieux. Pourquoi?

L'on s'est demandé plus haut si l'art n'était pas ontologiquement réfractaire au questionnement sociologique. Revenons sur cette question, que les sociologues de l'art actuels n'hésitent parfois pas à pointer, mais écartons d'abord l'hypothèse selon laquelle les préoccupations esthétiques ne seraient qu' anecdotiques et sans intérêt chez les fondateurs de la discipline. En raison de son caractère de fait marginal, la tentation serait forte de considérer que la mise en évidence de l'existence (ou de l'exhumation?) d'une sociologie de l'art chez les fondateurs ne consiste qu'en une entreprise de réhabilitation, de légitimation ou encore de revalorisation de la sociologie de l'art, dont on sait qu'elle traite d'un objet certes noble, mais socialement peu utile. C'est cette ambivalence de l'objet que Marcel Fournier (2003) relève lorsqu'il dit de la sociologie de l'art qu'elle "se retrouve au sein de la sociologie dans une position paradoxale: à la fois inférieure et supérieure, à la fois dominante et dominée, pour prendre des expressions chères à Pierre Bourdieu”. Elle est en effet, selon les termes de son analyse, "supérieure par son objet", socialement valorisé, noble, luxueux et distinctif. Dans le même temps, elle est " inférieure par la place qui est accordée à la discipline, et aussi par les positions qu'occupent dans le champ ceux qui s'y intéressent ". ${ }^{22}$ C'est à travers cette ambiguïté que la spécialité est également présentée dans un numéro spécial de la Revue française de sociologie ${ }^{23}$ qui lui est consacré; elle y est, dans le texte introductif, présentée comme “Parente pauvre mais noble (trop ?) de la discipline". ${ }^{24}$ Le sociologue Jean-Claude Chamboredon (1986, p. 505) se demande d'ailleurs, non sans une certaine ironie sans doute, ce que peut bien peser " la peinture de Courbet ou la forme des drames d'Ibsen au regard des perspectives de mobilité sociale ou de l'évolution $d u$ groupe domestique?". Cette hypothèse concernant la survalorisation des préoccupations esthétiques serait d'autant plus plausible que la mise en évidence d'une sociologie de l'art des origines est de façon quasi-systématique l'œuvre des sociologues de l'art eux- 
mêmes: ont été mobilisés dans la présente contribution les arguments de Pierre-Michel Menger ou de Marcel Fournier par exemple. Peutêtre pourrait-on concéder à cette hypothèse qu'il faudrait nuancer le propos lorsque celui-ci insiste sur le caractère central et essentiel des études esthétiques.

Cependant, à cette hypothèse sociologique, nous préférons l'hypothèse épistémologique déjà évoquée selon laquelle " l'exigence de singularité "25 de l'art pose problème à l'analyse sociologique, et ce, depuis les origines de la discipline, en raison de ses fondements mêmes et de ses ambitions. Cette seconde hypothèse présente l'avantage de rendre compte à la fois de la volonté de prendre en charge la question artistique (liée à l'impérialisme sociologique que nous avons évoqué et au défi que pouvait constituer cette approche) et des difficultés de la sociologie à se confronter à un tel objet. Ces difficultés ne se donnent pas à voir uniquement dans l'absence de discours unifié sur l'objet dans la revue étudiée. Elles apparaissent à différents moments de la discipline, ce dont la première hypothèse ne permet pas de rendre compte.

Selon Pierre-Michel Menger, le défi lancé à la sociologie naissante et le problème rencontré par Durkheim s'expriment sous la forme de la question suivante:

Comment s'accordent le fait de l'extrême différenciation de la production artistique, où s'exprime l'individualisme dont l'artiste est réputé incarner la forme accomplie, et la dimension collective, communautaire, de l'imputation de valeur à des symboles et à des œuvres engendrés par une telle dynamique sociale ? Comment, en d'autres termes, rendre compte simultanément de la singularité des œuvres et de leur condition de production, d'une part, et de la généralité ou de l'universalité de leur signification et du plaisir esthétique qu'elles procurent, d'autre part? (Menger, 2001, p. 314)

Pour le sociologue, l' antinomie classique se transforme en une tension entre individualisation des actes de création et " universalité de la valorisation des euvres reconnues", et ne trouve pas de résolution satisfaisante chez Durkheim. Cette contradiction semble s'inscrire dans le cadre plus large du problème de la saisie de l'art 
par la discipline sociologique, problème auquel les acteurs ont tout au long du siècle répondu de diverses façons. Il serait en effet possible d'envisager les différentes approches en sociologie de l'art comme autant de manières de dépasser cette contradiction apparente. C'est de cette manière qu'il est possible d'interpréter, dans ses déclinaisons contemporaines, l'étude de l'art au prisme de son marché initiée par Raymonde Moulin dès la fin des années 1950 en France. Car c'est précisément parce que lui sont associés deux valeurs antinomiques, que sont l'exigence de singularité et l'exigence d'universalité que "l'art permet, plus que tout objet, de repenser, et parfois même d'abandonner ou de renverser, un certain nombre de postures, de routines, d'habitudes mentales ancrées dans la tradition sociologique" (Heinich, 1998, p.8). 


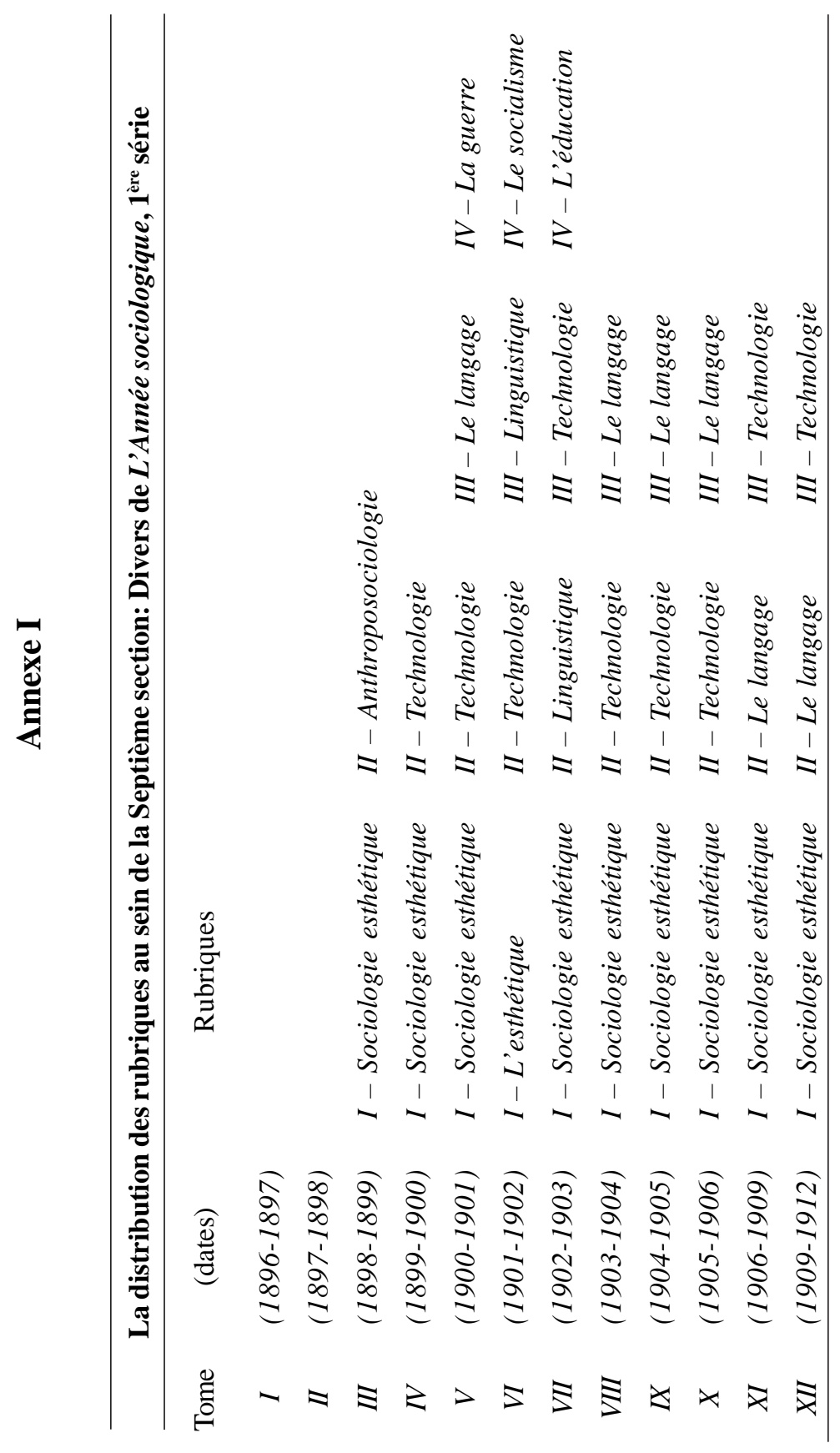




\section{Notas:}

1 L'on peut citer à titre d'exemples Collins (1988), ou encore en France, les travaux initiés par Pierre Bourdieu présentés de manière systématique dans Science de la science et réflexivité (2001).

2 Même si il nous importe d'affirmer que ces réductions sont également liées aux exigences épistémologiques de cet objet: de fait, "une discipline scientifique ne retient de la réalité que ce que l'ensemble de ses moyens théoriques et pratiques permet d'investiguer. Elle opère de cette manière une réduction méthodologique de la réalité" (Franck, 1999, p. 129).

3 De la même façon et de manière récurrente surgissent également des velléités interdisciplinaires. À ce sujet, voir Valade (1999).

4 Le premier volume de la revue verra le jour en 1898. Voir annexe I.5

5 Karady (1979) appelle légitimité institutionnelle «la reconnaissance accordée à une formation à ambition scientifique par les institutions qui se situent au sommet de la hiérarchie socialement organisée de la consécration, de l'inculcation, de la production, de la reproduction, de la conservation et de la diffusion des connaissances» (p. 49); la légitimité proprement scientifique «a pour siège l'opinion savante tout entière, qu'elle s'exprime ou non au travers des institutions» (p. 50).

6 Àce sujet, voir Mucchielli, 1998, p. 18-19.

7 Cuin et Gresle, 1992, p. 80.

8 Voir annexe 1.

9 "L'Année sociologique et la sociologie "esthétique", International Sociology of Art Conference (Ecole nationale supérieure des Beaux Arts - Ecole Nationale des Ponts et Chaussées), avril 2003.

10 Heinichn, 2001, p. 10.

11 Gummeref, B. The Beginnings of Poetry. New York: Macmillan, 1901, L'Année sociologique, 1 ère rie, sixième année, p. 560.

12 L'Année sociologique, $1^{\text {ère }}$ série, quatrième année, 1901, p. 588-591.

13 Roth, W.-E. Games, Sports and amusement. L'Année sociologique, $1^{\text {ère }}$ série, septième année, p. 666.

14 L'Année sociologique, $1^{\text {ère }}$ série, quatrième année, 1901, p. 584.

15 Op. cit.

16 Baratono, A. Sociologia estetica, L’Année sociologique, $1^{\text {ère }}$ série, troisième année, p. 576-577. 
17 Parodid, D., op. cit., p. 577.

18 Parodi, D. L’Année sociologique, $4^{\mathrm{ème}}$ année, p. 588.

19 Mauss, M. L’Année sociologique, $6^{\text {ème }}$ année, p. 560.

20 Chaillie, R. L’Année sociologique, $9^{\text {ème }}$ année, p. 588.

21 Karadi, V., op. cit.

22 Concernant ce deuxième point, Marcel Fournier note cependant que cette marginalité des sociologues de 'art est toute relative et qu'elle est souvent exagérée par ceux-là mêmes qui la pratiquent.

23 Sociologie de l'art et de la littérature. Revue française de sociologie, v. 27. 1986.

24 Chamboredon, J.-C.; Menger, P. -M. Présentation, Revue Française de Sociologie, v. 27, 1986. p. 363

27 Nous empruntons l'expression à la sociologue de l'art Nathalie Heinich.

Resumo: Quando a sociologia das ciências se apossou da sociologia da arte

Se, classicamente, a sociologia das ciências é vista como o estudo das atividades relevantes das ciências da natureza, é dentro no quadro de uma sociologia das ciências humanas e sociais que desejamos inserir nossas próprias pesquisas, e estudar a constituição da sociologiaa da arte na França, como subdisciplina distinta de outros campos de investigação da sociologia e distinto das outras disciplinas que, tradicionalmente, se ocupavam do seu objeto: a estética e a história da arte. Abordaremos o primeiro momento do processo de "disciplinarização" da sociologia da arte, isto é, sua inserção no projeto durkheimiano.

Palavras-chave: sociologia das ciências, história da sociologia, disciplina, disciplinarização, comunidade, Anné sociologique, Emile Durkheim.

\section{Bibliographie}

BARATONO, A. Sociologia estetica. L'Année Sociologique, $1^{\text {ère }}$ série, troisième année, p. 576-577, 1898-1899.

BESNARD, Ph. La formation de l'équipe de l'Année Sociologique. Revue Française de Sociologie, v. 20, n. 1, 1979. 
BOURDIEU, P. Mais qui a créé les créateurs? In: Questions de Sociologie. Paris: Minuit, 1980.

BOURDIEU, P. Science de la science et réflexivité. Paris: Raisons D'agir, 2001.

CHAMBOREDON, J.-C. Production symbolique et formes sociales. De la sociologie de l'art et de la littérature à la sociologie de la culture. Revue Française de Sociologie, v. 27, 1986.

CLARK, T. N. The structure and functions of a research institute: The Année Sociologique. Archives Européennes de Sociologie, v. 9, n. 1, p. 7291, 1968.

COLLINS, R. The Sociology of Philosophies: a global theory of intellectual change. Cambridge Harvard University Press, 1988.

CUIN, C.-H.; GRESLE, F. Histoire de la sociologie - vol. I : avant 1918. Paris: La Découverte, 1992.

FOURNIER, M. Durkheim, L'Année Sociologique et l'art. Etudes durkheimiennes, n. 12, janvier, 1987.

Les hauts et les bas d'une diva. Sociology of Art Conference, Paris, avril, 2003.

FRANCK, R. La pluralité des disciplines, l'unité du savoir et les connaissances ordinaires. Sociologie et Sociétés, v. 31, n. 1, 1999.

HEINICH, N. Du peintre à l'artiste: artisans et académiciens à l'âge classique. Paris: Minuit, 1993.

. Ce que l'art fait à la sociologie. Paris: Minuit, 1998.

. La sociologie de l'art. Paris: La Découverte, 2001.

HENNION, A. La sociologie est-elle définitivement allergique au génie? Pour un programme constructiviste fort en sociologie de l'art. In: BERTRAND-DORLEAC, L.; GERVEREAU, L..; GUILBAUT, S.; MONNIER, G. (Ed.). Où va l'histoire de l'art contemporain? Paris: L'Image \& ENSBA, 1997.

KARADY, V. Stratégies de réussite et modes de faire-valoir de la sociologie chez les durkheimiens. Revue Française de Sociologie, v. 20, n.1, 1979.

MAUSS, M. Divisions et proportions des divisions de la sociologie. L'Année Sociologique, nouvelle série, 2, 1927. Repris in MAUSS, M. Euvres, t. 3: Cohésion sociale et divisions de la sociologie. Paris: Minuit, 1969. 
MENGER, P.-M. Durkheim et la question de l'art. In: FABIANI, J.-L. (Dir.). Le goût de l'enquête - Pour Jean-Claude Passeron. Paris: L'Harmattan, 2001.

MUCCHIELLI, L. La Découverte du social: naissance de la sociologie en France. Paris: La Découverte, 1998.

Sociologie de l'art et de la littérature. Revue Française de Sociologie, v. 27, 1986.

TIRYAKIAN, E.-A. L'école durkheimienne à la recherche de la société perdue : la sociologie naissante et son milieu culturel. Cahiers Internationaux de Sociologie, v. 66, 1979.

VALADE, B. Le "sujet" de l'interdisciplinarité. Sociologie et Sociétés, v. 31, n. 1, printemps 1999. 